\title{
INTERVIEW WITH VANESA ENRÍQUEZ RAÍDO, AUCKLAND UNIVERSITY, NEW ZEALAND
}

\section{By Carmen Valero-Garcés}

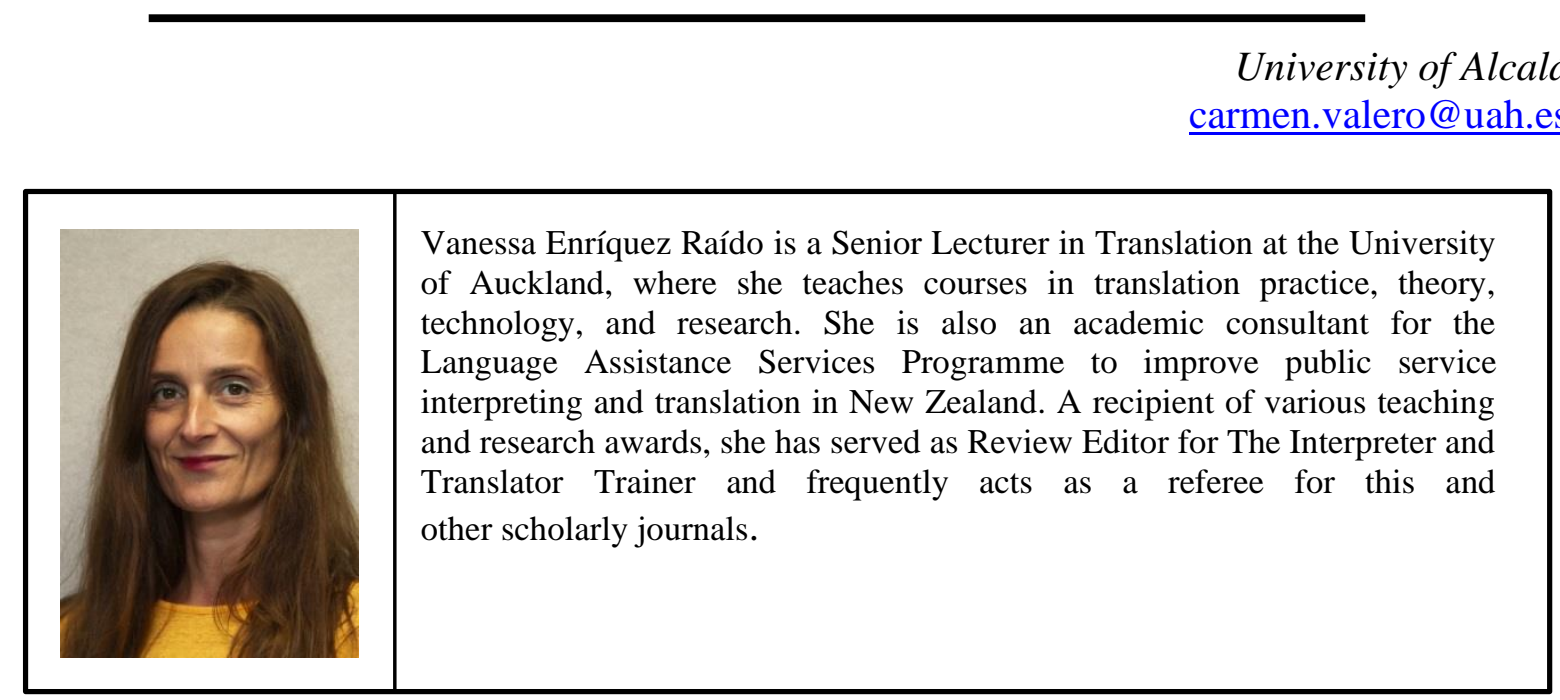

C.V.G. As an expert in research methodology, do you see a significant advance in $T \& I$ research in relation to other broader areas such as Linguistics? What about T\&I and PSIT?

V.E.R. The growing number of publications, seminars, and workshops related to research methods in translation and interpreting studies (TIS) testifies to a notable interest and progress in the empirical study of translation and interpreting (T\&I) phenomena. Unlike other, less ill-defined knowledge areas, research advances in TIS tend to mirror paradigmatic shifts in neighboring fields such as applied linguistics, writing studies, ergonomics, brain research, etc. One of the most significant developments in our area has been the move away from the early prescriptive and anecdotal accounts of T\&I as a product to the empirical examination of related phenomena as a process, and towards increased scientific rigor. The evolving and interdisciplinary nature of T\&I thus entails that our field of inquiry is often in a state of flux, with researchers adopting new methods, research designs, and data collection tools to address a wide range of questions, thereby bringing innovative change.

The gamut of possibilities afforded by technology also means that we have a larger pool of more sophisticated tools and methods to probe deeper into process and workflow-related inquiries - e.g., via eye tracking, screen/video/audio recording, keystroke logging, electroencephalography (EGG) and functional magnetic resonance imaging (fMRI) - as well as product-oriented questions, most notably through corpus linguistics tools. Technology developments have also led to research advances in PSIT, albeit perhaps more in terms of new themes and refined research foci-e.g., the impact of communication technologies such as videoconferencing on different settings of video remote interpreting - than from a purely 
methodological perspective. In this respect, the methods and data gathering tools used in PSIT do not seem to differ greatly from those used in other areas of TIS.

C.V.G. In your opinion, what are the most common research methods in Translation and Interpretation Studies? And in the specific case of PSIT? Do you see any differences or preferences?

V.E.R. The interest in more scientific descriptions of $T \& I$ phenomena has brought about a remarkable increase in the use of quantitative research methods and tools such as those mentioned above, including corpus tools. Given the proliferation of quantitative data generated by said tools and the large data sets available from different digital sources (e.g., Web, social media and crowdsourcing), there is a justifiable preference for statistical methods to analyze and interpret said data. In PSIT, a field that is firmly grounded in the societies and communities we live in, qualitative research methods like ethnography and interviewing tend to prevail over quantitative ones, although a mixed-method quantitative and qualitative approach is as frequently adopted in PSIT as it is in TIS at large. The same applies to multi-method approaches, which typically fall under the quantitative or qualitative paradigm. What seems to be a distinctive and invariable feature of PSIT research is the need for a multi-participant or multi-stakeholder approach involving different key agents in the investigation process. This is largely so because PSIT cuts across the legal, economic, political, cultural, and public dimensions of our respective countries, societies, and target populations, thereby focusing on their beliefs, attitudes, norms, practices, standards and policies. Much research is therefore interdisciplinary, context-dependent and small-scale, as it tends to address specific settings, institutions, providers, and users, although cross-border research via large-scale projects is also becoming increasingly popular.

C.V.G. The fact that scientific literature increasingly offers us more specific publications on research methods in the manuals and encyclopedias of the T\&I area -including monographs - indicates a growing interest in research, but has progress been made in the systematic analysis of data at the PSIT? What about in the use of large databases? Are there still many case studies? Why?

V.E.R. The surge of (non-)forced immigration and other global flows leading to the growing recognition of PSIT as a prominent field of study has been a catalyst for its examination in more systematic and reflective ways. Notwithstanding this new prominence, the specificity and inherent challenges of PSIT research (e.g., sampling, participant recruitment, ethics approval, informed consent, as well as cross-cultural validation of questionnaires) pose significant obstacles to systematic data analysis and the generalization of findings to larger populations. While the latter is the ultimate goal of quantitative research, in PSIT the extent of confounding variables spanning differing socio-political, socio-cultural, and sociolinguistic contexts entails the need to generalize to limited and specific target populations only. Like TIS in general, this phenomenon tends to complicate replicability and systematic analysis, among others. It is not surprising then that PSIT researchers tend to favor in-depth qualitative research to identify patterns that they can subsequently compare across different demographic contexts, for example, through bibliometric studies. In fact, the specificity of PSIT encounters can substantially explain the prevalence of qualitative methods such as case studies, (non-) participant observation, ethnography, action research, and survey research via interviews (individual and focus-group) and personal narratives. The development of 
databases that can serve as repositories of recorded interactions (e.g., triadic interactions in interpreter-mediated medical consultations) would certainly be a fruitful avenue for systematic quantitative and qualitative analyses of stakeholder encounters using a discourseanalysis approach, among others. The same is true for the systematic study of policy documents through corpus-based studies.

\section{C.V.G. What are the trends in translation theory? Has functionalism been overcome? What about constructionist theories? And the sociological or ethnographic approaches?}

V.E.R. Different theoretical models will always influence - explicitly or implicitly- the ways in which individuals understand the notion of translation and how they approach it. Nevertheless, well-known constructs based on equivalence and functionalism-e.g., intended audience, translation purpose, end-users, accuracy, adequacy, acceptability, and usabilitycontinue to govern not only much of today's translator education and scholarly debate, but also the notion of quality in a variety of industry settings. The "fit-for-purpose" model that underpins niche applications of machine translation (MT) and related automatic and human metrics of post-edited quality represent, for example, direct applications of functionalist approaches to translation. The emergence of new crowdsourcing practices and workflows that are used to scale translation within certain industry sectors has also contributed to the consolidation of the concept of fit-for-purpose translation as a service that can be fine-tuned according to various project specifications, especially in the context of industry standards and translation quality evaluation.

In terms of other theories or approaches, PSIT scholars and researchers can hardly do without sociology or ethnography, for instance, at least not to some extent, due to the deepseated societal roots of language assistance services in the public sector.

\section{C.V.G. In your opinion, what are the trends in the PSIT?}

V.E.R. Similar to other fields undergoing development, some of the trends that we can observe in PSIT include, but are not limited to:

- The quest for legitimization and increased recognition and visibility among key stakeholders and other social agents.

- The professionalization of non-professionals, above all in the context of languages of low diffusion and low resources.

- Ethical dimensions, competences, and standards associated with the use of both professionals and non-professionals across different settings and for specific target populations.

- Increased hybridization of professional roles, availability of bilinguals, and cost concerns.

- The use of tools and technologies that can support multilingual service encounters.

- The development of resources such as guidelines and online repositories for public access and awareness-raising among stakeholders.

- The establishment of international networks and associations, as well as the development of training and certification schemes.

- Policy development, ideology, etc. 


\section{C.V.G. What are the main trends in pedagogy or didactics of T\&I? Is the same observed in relation to the PSIT?}

V.E.R. The ongoing impact of multiple language and translation technologies on contemporary $T \& I$ leads to many $T \& I$ training programs worldwide adopting a technologyoriented curriculum. With recent developments in natural language processing and the adoption of neural approaches to T\&I-oriented productivity tools such as MT, automatic speech recognition, and automatic term extraction, the emphasis appears to lie on the training of proficient and critical users of a wealth of technologies and tools available to them. Educating T\&I students to become consultants advising on language services across the full spectrum of machine and human translation solutions seems to be another common trend in the age of MT. Here, like in other spheres of TIS, including PSIT, we continue to encounter a mixture of teaching and learning methods and approaches such as project and task-based learning, discovery learning, situated learning, (semi-) authentic practice, etc.

Furthermore, the exponential growth of digital content requiring large-scale translation services at low cost and faster speed suggests that the ability to post-edit raw MT to different quality levels is likely to find its place in contemporary competence models, i.e., not just in industry standards that apply to specific market segments. Also, given that current business translation practices increasingly involve both professionals and non-professionals in emerging crowd-based workflows, enabling students to familiarize themselves with new translation quality assessment (TQA) methods and models -beyond inbuilt and standalone automatic quality assurance $(Q A)$ or quality control $(Q C)$ tools - is likely become another popular trend. The present industry shift towards broader requirements for TQA will eventually correlate with training in the critical use of standard error typologies for human evaluation (including linguistic annotation of post-edited errors for MT performance), automatic metrics of MT evaluation, content profiling, and automatic MT quality estimation. As crowdsourced evaluation is also becoming increasingly popular within the MT market, particularly for large-scale translation services, T\&I trainees will benefit from learning how to assess and compare different quality types across multiple work scenarios, with crossfertilization from one TQA method to another. Quality issues also feature prominently in PSIT training scenarios, where additional pedagogical trends seem to correspond to the research trends identified earlier. It will be interesting to observe if and how new crowdsourcing models and online communities develop with reference to the provision of language assistance services across the public sector. It would certainly complement our existing knowledge about emergency and crisis scenarios allowing us to harness the power of the crowd and leverage the communication technologies, tools, and resources at our disposal to bridge communication gaps.

\section{C.V.G. Are there any policy changes in T\&I practice? What about PSIT?}

V.E.R. My impression is that policies concerning language assistance services (especially state-funded language support) are still strongly rooted in human rights-based discourses that cut across a number of common themes such as equal access, social inclusion, health and social well-being, language proficiency, political participation, etc. The rise in crossborder migration and global mobility has been causing a shift towards politics of recognition 
and diversity in certain geonational contexts. Regardless of the type of policy approach used to address and eventually provide language support at a national level, significant differences in political systems and the availability of public resources continue to affect the development and actual implementation of international legislation regulating the provision of translators and interpreters in the public sector. Although statutory obligations that specifically mention the right to interpreting and translation in a legal context do exist at a supranational level, especially in the area of criminal proceedings (e.g., Directive 2010/64/EU of the European Parliament), the lack of explicit international legislation guaranteeing the right to interpreting in healthcare settings, for example, may be seen as an outlier in the field of Human Rights. As Bradely Dalton-Oates (2017: 231) notes: "It is one thing to 'strengthen cooperation between Member States' [ergo, legal translation and interpreting), and quite another to issue prescriptive and binding rules as to the necessary behavior between a State and an individual [e.g., medical translation and interpreting]." New Zealand (my immediate context) is one of those nations that grants the right to interpreting in healthcare settings. However, unlike in the European context, where said right appears to be at the Centre of language policies and language management, in New Zealand, a country that has no official language policy, the legal right to health interpreting emerged from a government inquiry into a large-scale cervical cancer research project (Ministry of Health 1988) that revealed doctors' unethical practices (see below).

\section{C.V.G. How is the emergence of technology in translation in general affecting research? What about PSIT in lesser-used languages?}

V.E.R. The shift towards hybrid crowdsourcing models and enhanced automation solutions to provide cost-effective multilingual services at scale demands systematic research into the use of aggregate data to translate, validate, and evaluate the input from very diverse crowds. Issues pertaining to inter-annotator agreement, for example, seem to drive current industry research involving novel approaches to managing crowdbased translation workflows inhouse. Yet, confidentiality and increased market competition, among other factors, tend to make this kind of research particularly challenging, despite the existence of non-disclosure agreements between industry and research partners. Translator-computer interactions and software usability aspects also necessitate ongoing research, just as they do in PSIT with regard to designing tools (such as mobile applications) that support and give feedback to a variety of language service providers. Although language technologies do not seem to be frequently used by public institutions - something that is often discouraged in related policies and guidelines - technology-enabled multilingual communication can be expected to rise sharply. This particularly applies to situations where professional public service interpreters are needed, yet not readily available. Video-mediated interpreting and other forms of remote interpreting such as telephone interpreting are being increasingly used to overcome this obstacle. Also, the ongoing development of MT systems and other automatic translation tools will eventually offer rare languages the resources they need. Further technology developments can also be expected to provide the PSIT community (and society at large) with bridging functions that can potentially support direct, i.e., unmediated, multilingual communication between service providers and their users. 


\section{C.V.G. How is it (or will it be) PSIT affected by open access and open research?}

V.E.R. The benefits of open access are well-known to researchers and institutions working across various disciplines. These benefits include larger audiences, faster publication cycles, more collaborators, and potentially more author citations, as well as increased recognition and research transparency. In the case of PSIT, open access can be especially beneficial to countries in the Global South, not only for researchers and institutions who cannot afford journal subscriptions, but also to create greater access opportunities (and hence growing awareness) for specific target populations and social actors who need language support to navigate decision-making in the public arena.

\section{C.V.G. How to assess the quality of research in PSIT?}

V.E.R. This can be achieved by using the same criteria to assess scientific rigor across quantitative and qualitative research. Although the epistemological and ontological positions (and hence their terminology) vary between these two paradigms, the criteria used to ensure robust research designs typically revolve around different types of validity, reliability and replicability, among others.

\section{C.V.G. What level of interdisciplinarity is observed in recent research?}

V.E.R. The very interdisciplinary nature of TIS means that establishing a definitive academic focus in our field is not easy, if not impossible or desirable. As already mentioned, PSIT cuts across the legal, economic, political, cultural, and public spheres of our countries, societies, and communities, thereby requiring an interdisciplinary approach to research. PSIT investigators usually engage in interdisciplinary research by drawing on sociological, political, and phycological approaches, among others. My perception is that more transdisciplinary research is needed to create specialized knowledge based on the participation of and closer collaboration among multiple researchers from various disciplines like medicine, public health, cross-border migration, etc.

\section{C.V.G. How to teach research method to young researchers in multilingual and multicultural contexts of minority languages?}

V.E.R. This could be done by creating training opportunities that are attuned to research needs of young researchers, also taking into consideration the local contexts in which they operate. Regardless of the specificity of different research contexts, I find that it is generally a good idea to train for all main stages of any research project, i.e., for multiple ways of collecting, processing, and analyzing data. I have also found throughout the years that discussing epistemological and ontological aspects of research - and linking these to suitable research designs - as well as learning how to conduct a thorough literature review and develop research assessment skills are fundamental components of any research training module. 


\section{C.V.G. What role do ethics play in research methods at PSIT?}

V.E.R. Research ethics is an imperative for any kind of investigation. In PSIT, it becomes even more relevant as ethics approval and informed consent are generally required from the organizations and participants under study. Cutting corners in terms of research ethics can have detrimental consequences for all parties involved. The above-mentioned New Zealand government inquiry into a large-scale cervical cancer research project, known as 'The Cartwright Inquiry 1988', revealed doctors' unethical practices in relation to communication, information sharing, and obtaining informed consent from women who did not have English as their first language. It also highlighted issues around the use of unqualified interpreters, such as relatives (mainly children) and untrained bilingual staff. On a more positive note, the inquiry led to several recommendations, including the development of a legislated Code of Patients' Rights and the establishment of an independent Health Commissioner.

C.V.G. What do you think of action research and the role of translators and interpreters in PSIT research?

V.E.R. The pros and cons of action research, a paradigm that originated in the field of education, are the same for any researcher across any discipline. Whereas this paradigm can often be the only means for PSIT investigators to access research participants - and indeed provide a suitable method for bringing about change and improving the efficiency of service delivery in a given sector - action researchers who also happen to be PSIT practitioners need to take extra care to avoid coercion and voluntary selection of participants, for example, while also ensuring honesty and research transparency.

C.V.G. In your opinion, what are the most effective methods to ensure the scientific, social and political impact of PSIT research?

V.E.R. Unfortunately, the growing recognition and legitimization of PSIT as a field of study has had limited impact on policymakers and other social actors involved in the provision and use of language support services across the public sector. It is still often the case that political discourses surrounding T\&I policies invariably tend to emphasize cost concerns, which suggests that large-scale and transdisciplinary studies of cost-effectiveness may have substantive impact on future policy development. Similarly, ongoing efforts to highlight the negative consequences that using untrained bilinguals can sometimes have among specific target populations and service providers are likely to influence policymaking to some extent.

C.V.G. Do you consider that research is essential for the professionalization of PSIT? How long does it take for PSIT to reach that level?

V.E.R. The key literature in PSIT tends to acknowledge that research and other disciplinaryled discourses have also had limited impact on professionalization so far. Nevertheless, efforts to address this challenge and bring about change appear somewhat more feasible and 
less complex in certain contexts than efforts aimed at influencing national policymaking -although this is also very much dependent on geonational contexts.

\section{Thank you}

\section{References}

Dalton-Oates, Bradley. 2017. "Medical translation: the neglected human right". International Journal of Human Rights in Healthcare, 10 (4): 228-238.

European Parliament. 2010. Directive 2010/64/EU of the European Parliament and of the Council of 20 October 2010 on the right to interpretation and translation in criminal proceedings. [Available at:

https://eur-lex.europa.eu/legal-content/EN/TXT/?uri=celex\%3A32010L0064]

Ministry of Health (New Zealand). 1988. The Cartwright Inquiry 1988. [Available at: https://www.nsu.govt.nz/health-professionals/national-cervical-screeningprogramme/legislation/cervical-screening-inquiry-0]

How to cite this article?/ ¿Cómo citar este artículo?

Valero-Garcés, Carmen (2020) "Interview with Vanesa Enríquez Raído, Auckland University, New Zealand". FITISPos-International Journal, 7 (1). 138-145. 